\title{
Passion and commensurability: both are needed for a concurrence of surgery and research
}

\author{
W. E. Thasler • A. Kleespies $\cdot$ R. Thasler $\cdot$ K.-W. Jauch
}

Received: 15 March 2013 / Accepted: 18 March 2013

(C) Springer-Verlag Berlin Heidelberg 2013

As motto for this year's 130th Annual Congress of the German Surgical Society (30th April to 3rd May 2013), we chose "Chirurgie mit Leidenschaft und Augenmaß". It expresses the demand of surgery for passion ("Leidenschaft"), which manifests in ardent craftsmanship and readiness towards patients, as well as "a sense of proportion" (“Augenmaß”), meaning commensurability, since every surgical treatment involves injury and cost. This dilemma is well effective in a surgeon's daily work, where he has to balance passionate surgical performance for the best of patients, who seek help and are dependant and trustful, with rational thought on which treatment is reasonable and feasible.

Continuing this year, four categories - one for each daywill structure a broad range of topics where such a balance in surgical decision making is critical: on Tuesday, talks and discussions will be focused on the treatment of elderly patients; the Wednesday sessions will cover the forming of working environments and efficient training for surgery; making and evaluation of innovation will be discussed in the sessions of Thursday and finally, Friday sessions will be focused on risk assessment, patient safety and quality assurance.

\section{Scientific topics}

Introducing last year's congress issue, Schneider at al. pointed out that translational science can form a "cycle of success" around the exploration of ideas in basic science, if it starts with a clinical problem and reaches out to a clinical application and

W. E. Thasler $(\bowtie) \cdot$ A. Kleespies $\cdot$ R. Thasler $\cdot$ K.-W. Jauch Department of General, Visceral, Transplantation, Vascular and Thoracic Surgery, Ludwig Maximilians University Munich, Marchioninistr. 15,

81377 Munich, Bavaria, Germany

e-mail: wolfgang.thasler@med.uni-muenchen.de its evaluation [1]. It is evident that the "surgeon-scientist" is best fitted to turn this cycle on and keep it going. However, Menger et al. stated in the same issue that commitment to research by surgeons is hampered by the increasing demand for other competences such as management and organisation. These skills are becoming increasingly important as the health system is changed by the use of economic tools, riveted on cost efficiency in patient care [2]. Since commitment of surgeons to research has to parallel their training and clinical qualification, this points towards a growing problem of balance between "passion", which "has been the immediate resource of discipline and methodical exploration in the life of great surgeons" [3] and commensurability.

Against this background, this issue of Langenbeck's Archives of Surgery presents scientific review articles on specific topics which will be discussed at the 130th annual conference of the German Surgical Society. They give special insights into two totally different issues, both highlighting the interdependence of surgery and research: Thasler et al. show that passion for research in surgery is needed especially in the field of translational research and its main methodological prerequisite: biobanking. However, a "sense of proportion" has to be also fostered with a view to patient rights and ethics, along with the need for external governance and interdisciplinary cooperation [4]. Metzger et al. give an overview on the development and perspectives of navigation as well as information technology in surgery from the perspective of a medical device provider. This article shows how the passion of surgeons drives clinical innovations, which make treatments - as a kind of perfect match with commensurability as stated above-more effective, reasonable or feasible [5].

Additionally, three further original articles focus the major issue of surgery in the elderly and senescence addressed at the conference on Tuesday, April 30th. This topic will gain 
tremendous importance within our surgical reality in the near future. Yamashita et al. can show that advanced age alone (older than 75 years) should not discourage surgeons from offering pancreatoduodenectomy (PD) in case of a periampullary tumour, since no significant difference in postoperative morbidity and perioperative mortality was found in their limited series of patients [6]. Oguro et al., however, present a higher incidence of severe postoperative complications after PD (> grade III, according to ClavienDindo classification) in their large group of octogenarian patients and conclude that elderly patients must be carefully selected for surgery [7]. Finally, Miccoli et al. point out that in thyroid surgery for benign disease not only feasibility and safety of the surgical procedure but also costs should be taken into account. In addition, a significant increase of costs must be calculated for extended preoperative cardiopulmonary evaluation and perioperative risk assessment in octogenarians [8].

All abstracts accepted for oral presentations in the field of surgical research to be presented in sessions dedicated to research are also included in this issue. Surgical research topics were presented by $25 \%$ of the overall abstract submissions. The rate of acceptance was about $40 \%$. For the first time, abstracts were not only chosen for dedicated research sessions: 55 research abstracts were additionally accepted as complementary parts of clinical sessions of the expert disciplines. Furthermore, about 80 abstracts were accepted for poster presentation. The research topic number 1 remains oncology with 33 (about $80 \%$ of accepted abstracts in total, including poster presentations), followed by transplantation research with 17 and biomarker research with 13 oral presentations. Besides other diverse issues ("Varia", 15), in total 18 abstracts cover the upcoming new topics: tissue engineering, regeneration/stem cell research and finally, as a still emerging discipline, biobanking.
In conclusion, these numerous high-quality contributions in the field of surgical research show that young surgeons still sustained a passion for research, which is promising for a future of surgery as an academic discipline and surgeons as scientists. However, the dispute on a still growing pressure also on academic surgeons as part of the health care system to primarily perform in measurements of cost effectiveness has to be continued.

Conflicts of interest None.

\section{References}

1. Schneider M, Welsch T, Kremer M, Büchler MW (2012) A cycle of success: the interdependence of surgery and science. Langenbeck's Arch Surg 397:493-494

2. Menger M, Schilling M, Schäfers H, Pohlemann T, Laschke M (2012) How to ensure the survival of the surgeon-scientist? The Homburg Program. Langenbeck's Arch Surg 397:619-622

3. Toledo-Pereyra LH (2002) Passion for surgical research. J Invest Surg 15(1):1-2

4. Thasler WE, Thasler R, Schelcher C, Jauch KW (2013) Biobanking for research in surgery: are surgeons in charge for advancing translational research or mere assistants in biomaterial and data preservation? Langenbeck's Arch Surg 398:487-499

5. Mezger U, Jendrewski C, Bartels M (2013) Navigation in surgery. Langenbeck's Arch Surg 398:501-514

6. Yamashita Y, Shirabe K, Tsujita E, Takeishi K, Ikeda T, Yoshizumi T, Furukawa Y, Ishida T, Maehara Y (2013) Surgical outcomes of pancreaticoduodenectomy for periampullary tumors in elderly patients. Langenbeck's Arch Surg 398:539-545

7. Oguro S, Shimada K, Kishi Y, Nara S, Esaki M, Kosuge T (2013) Perioperative and long-term outcomes after pancreaticoduodenectomy in elderly patients 80 years of age and older. Langenbeck's Arch Surg 398:531-538

8. Miccoli P, Fregoli L, Rago R, Matteucci V, Miccoli M, Materazzi G (2013) Increased costs of perioperative risk assessment for thyroid surgery in elderly people (over 80 years) presenting a benign disease. Langenbeck's Arch Surg 398:525-530 